\title{
Foreword
}

In 2008, the National Institutes of Health hosted over five thousand scholars at its first Science of Eliminating Health Disparities Summit. The subsequent annual summits were designed to encourage integration of science, practice, and policy to build a healthier society. As the opening plenary keynote speaker for the first one, I was asked to address "Health Disparities and the Intersection of Science and Policy." I deliberately anchored my talk in an explanation of health disparities as a reflection of social stratification and inequitable resource allocations along "racial" and "ethnic" lines. But what do we mean by "racial" and "ethnic" lines?

The scholars in this volume challenge us to be more precise about how we define and operationalize this type of stratification. Why? Because individuals are born into our society that neither treats people nor distributes opportunity equally. We observe discrimination, poverty, and other forms of oppression play out at the community level affecting overall community environment and opportunities. Especially in resource-poor communities, the stresses of daily life bear down on the minds and bodies of residents, inviting illness through environmental exposures and other types of stress that are expressed biologically in what we recognize as disease.

While Congress has required documenting these "racial" and "ethnic" disparities annually by the Agency for Health Research and Quality, it has done little to remedy the situation. Over the last few years, private philanthropies, such as the Robert Wood Johnson Foundation and the California Endowment, have recognized the need to prevent disparities by addressing the inequities in our society as well as addressing health care disparities—creating a more just society and thus a more healthy nation.

Interdisciplinary research plays an increasingly important role in addressing highly complex social issues such as health disparities. It allows scholars to reach beyond the boundaries of their own disciplines and to adapt or develop new methods of analysis. Institutional policies that support integrating the social and biological sciences could represent the twenty-first-century breakthrough similar to what we experienced in the twentieth century when biological and chemical scientists developed the fields of biochemistry, molecular biology, and molecular genetics that led to the sequencing of the human genome. Supporting interdisciplinary health and social science investigations 
and the development of transdisciplinary fields requires our nation's funding institutions and universities to be more flexible and open to new ways of seeing things. The Institute for the Study of "Race” and Social Justice within the RWJF Center for Health Policy at the University of New Mexico is one example of such efforts.

Sequencing the human genome provided definitive proof that there is no genetic or biological evidence for the concept of "race." Yet in our society we often rely on assumptions about "race" that erroneously assume that it is interchangeable with genes to explain the world and our understanding of it. Scientists are not immune from using the myth of race as biology in dangerous ways. When I arrived in New Mexico in 2008, I had the opportunity to participate in the annual Cancer Center retreat. Researchers shared the marvelous molecular discoveries brought about by genomic research advances, which offer prospects for innovations in treatment for a wide variety of cancers; however, I was taken aback when colleagues resorted to explaining statistical propensities for groups of people in New Mexico using wildly inaccurate racial stereotypes. These genetic reductionist interpretations of health disparities denied American Indian spiritual beliefs, challenged the history of Hispanic settlement in the region, and posed findings in ways that could be misinterpreted in policy implementation.

Despite all the problems inherent in the erroneous assumptions associated with the concept of "race," it is imperative that we continue to collect data that captures the lived experiences and practices that contribute to the racialized inequality that is reproduced at all levels of society. While some may argue for a color-blind society and an end to the collection of "race" or "ethnic" data, this book deliberately understands "race" as a social construction that can be mapped vis-à-vis legal efforts to eliminate racism in all its forms-individual, institutional, and systemic. For example, in the areas of banking and housing we have largely eliminated public policies restricting groups of people from living in certain areas; however, informal practices in the mortgage and real estate industries continue to produce de facto segregation.

Over the last several decades, we have negligently accepted racial categories in our work without the typical scrutiny we use to define variables for our analyses. Instead of interrogating and capturing the social construction of race in our research, we have at times blindly treated race as if it were a static characteristic that allows for the comparison of the health of different groups in our society. Or we have misused race as a proxy for many unmeasured factors in our studies, for example, class or culture, thereby masking what may be amenable to clinical intervention or social policy intervention. 
It is important that a paradigm shift take place in health disparities research, and Mapping "Race" helps catalyze it. Researchers should depart from the premise that racial and ethnic identities are fluid in both governmental and social settings. In order to combat discrimination, in 1976 Congress created the term "Hispanic" to group together a population that differs enormously by history, nationality, social class, legal status, and generation in the United States; it has little or no meaning outside of the U.S. political context. As scholars, scientists, and policy makers, we must challenge ourselves to be more precise with our categories of analysis and interpretation. The subjective meaning of such labels and whether they are situationally asserted are open empirical questions that need to be investigated.

\section{R. Burciaga Valdez}

July 2012 
